\title{
Simulación del inversor multinivel de fuente común como variador de frecuencia para motores de inducción
}

\section{Simulation of a common source multilevel inverter as frequency variator for induction motors}

\author{
Luis David Pabón-Fernández ${ }^{1}$ \\ Jorge Luis Díaz-Rodríguez ${ }^{2}$ \\ Aldo Pardo-García ${ }^{3}$ \\ Recibido: diciembre 27 de 2015 \\ Aceptado: junio 28 de 2016
}

\begin{abstract}
Resumen
En el presente artículo se muestra la simulación de un convertidor multinivel trifásico de fuente común de 15 escalones por línea, como variador de frecuencia para la alimentación de un motor de inducción. Este variador de frecuencia adopta modulaciones optimizadas en los primeros 50 armónicos; el algoritmo de optimización fue desarrollado en Matlab. Los resultados de la simulación permiten validar los beneficios que tiene este tipo de alimentación del motor de inducción en cuanto a calidad de la energía, tanto en el voltaje como en la corriente.
\end{abstract}

Palabras clave: inversor multinivel, variación de frecuencia, motores de inducción, distorsión armónica.

\begin{abstract}
This paper describes the simulation of a threephase multilevel common source converter of 15-line levels, as variable frequency drive for fed induction motors. The variable frequency drive is optimized in the first 50 harmonic responses; the optimization algorithm was developed in Matlab. The simulation results validate the benefits of this kind of fed induction motor with regard of the power quality in both: voltage and electrical current behaviour.
\end{abstract}

Keywords: multilevel inverter, frequency variation, induction motor, harmonic distortion. 


\section{Introducción}

El control de velocidad de motores de inducción, por lo general, utiliza como elemento final de control un convertidor de potencia que permite variar la frecuencia de la tensión de alimentación, con el fin de variar la velocidad síncrona y por ende la velocidad de la máquina (Castro-Galeano, PintoSalamanca, \& Amaya-Quitián, 2014; Mora, 2005). Los variadores utilizados en estas aplicaciones, por lo general, son inversores convencionales que utilizan técnicas de modulación de ancho de pulso, PWM por sus siglas en inglés (AraqueGallardo, Díaz-Rodríguez, \& Guerrero, 2016; TorresBarahona, León-Medina, \& Torres-Díaz, 2012). Además, la técnica de control utilizada, como el control vectorial por campo orientado o el control escalar, dependerá de los requerimientos específicos de cada aplicación (Bermeo et al., 2016; Vas, 1998).

Sin embargo, estos convertidores tienen un problema en lo referente a la calidad de la energía (Díaz-Rodríguez, Pabón-Fernández, \& Torres-Chávez, 2012; Sánchez, 2009), ya que por sus formas de onda cuadrada, generan gran cantidad de contenido armónico que provoca problemas como calentamiento de los devanados y generación de pares parásitos opuestos (Yumurtaci, Vakkas-Üstün, Varbak-Neşe, \& Çimen, 2008).

En los controles de velocidad de motores de inducción, los convertidores deben, además de variar la frecuencia, controlarelvalor dela magnitud de la tensión eficaz, RMS por sus siglas en inglés, con el fin de que a bajas velocidades la máquina no se sature y provoque corrientes excesivas (Mora, 2005; Restrepo-Chaustre, Becerra-Vargas, \& Pardo-García, 2015); este control el variador lo realiza mediante el aumento o disminución de la duración de los pulsos (Fitzgerald, Kingsley, \& Umans, 2003; Pinzón-Ardila, 2015). Esto hace que la forma de onda de la tensión a la salida del convertidor dependa de la frecuencia y el valor de la tensión que se desea; de esta forma el THD, Total
Harmonic Distortion, dependerá de la tensión y frecuencia de la salida del variador de frecuencia; es decir, el THD será una función THD $(V, f)$ (Barbera, Mayer, \& Issouribehere, 2009).

En la actualidad, la implementación de convertidores de potencia multinivel es una alternativa para la reducción del contenido armónico a la salida de las etapas de inversión DC/AC (Araque-Gallardo, Díaz-Rodríguez, \& Gualdrón-Guerrero, 2013), como las requeridas en aplicaciones de energías alternativas, en equipos de descargas de alta tensión o en el control de velocidad de motores (Shimi, Tilak, \& Jagdish, 2016; Mora-Mendoza, Sarmiento-Santos, \& Casallas-Caicedo, 2014); esto debido a que el THD que presentan es mucho menor que el de los convertidores convencionales (Malinowski, Gopakumar, Rodríguez, \& Pérez, 2010; Pradeep \& Jaseerali, 2015; Taleba, Benyoucefa, Helaimia, \& Boudje, 2015). Sin embargo, a la hora de ser aplicados en el control de velocidad, las técnicas de modulación presentan el THD como función de la frecuencia deseada y la tensión (Manasa, Balaji-Ramakrishna, Madhura, \& Mohan, 2012), no llegándose a presentar un óptimo nivel de armónicos para todas las frecuencias.

Es por lo anterior que este trabajo busca validar, mediante la simulación, las ventajas de alimentar las máquinas de inducción con un convertidor de frecuencia variable de tipo multinivel de 9 escalones por fase, que permita tener una calidad de energía constante e independiente de la tensión y la frecuencia a la salida del convertidor, presentando así un espectro puro en la banda de los primeros 50 armónicos, independientemente de la tensión y la frecuencia que el control de velocidad requiera.

\section{Marco teórico y metodología}

\subsection{Topología del convertidor}

La topología de inversor multinivel seleccionada para la simulación fue el convertidor de puente $\mathrm{H}$ 
en cascada asimétrico de fuente común, con relación 1:3 de 2 etapas, el cual genera 9 niveles de tensión a la salida de cada fase; la diferencia de potencial de las tensiones de fase genera un voltaje de línea con 15 escalones. En la figura 1 se muestra la topología trifásica que no es más que el triplicado de una fase, como se puede observar las tres fases comparten el bus de DC (Pabón-Fernández, Díaz-Rodríguez, \& Pardo-García, 2016).

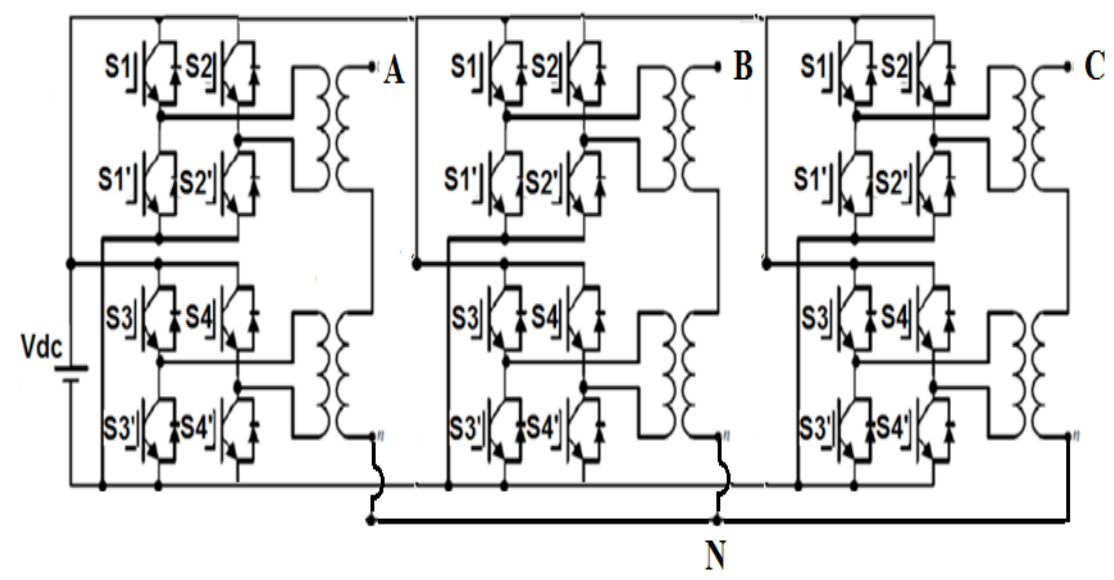

Figura 1. Topología del convertidor simulado.

\subsection{Aspectos generales de la simulación}

Para la simulación se utilizó un algoritmo programado en Simulink de Matlab; la programación de este código se realizó a través de bloques Matlab function, el cual se compila directamente en lenguaje C. Este algoritmo se encarga de recibir una dirección i, que el control de velocidad establece en dependencia de la tensión y frecuencia deseadas y obtiene la información de estados y el vector de tiempos de la modulación requerida. La figura 2 presenta el esquema del algoritmo de control del convertidor.

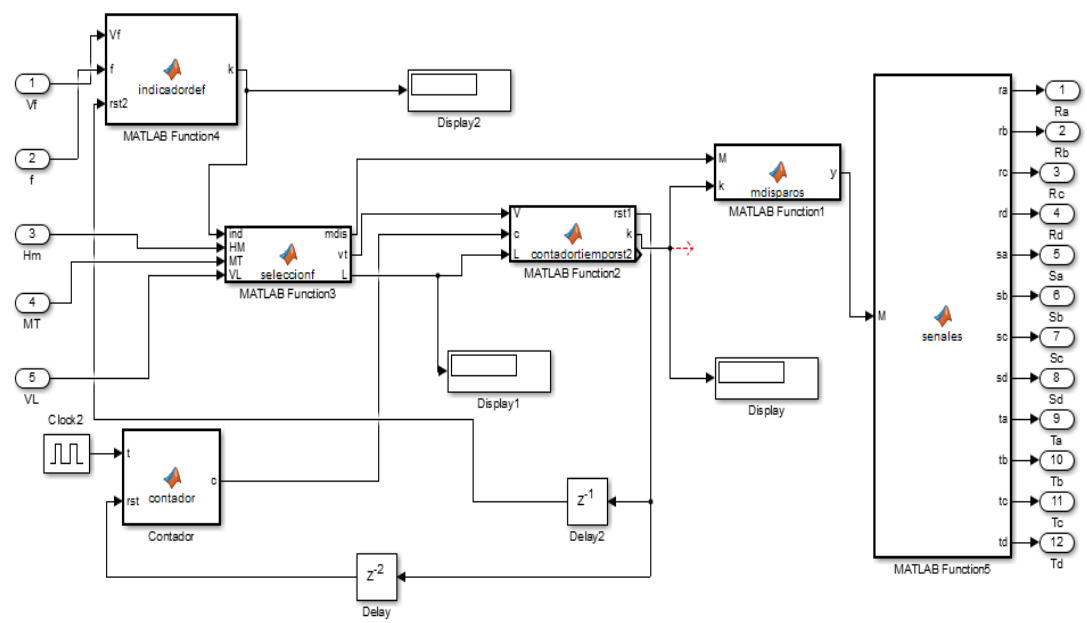

Figura 2. Algoritmo de control del convertidor de potencia. 
Este bloque recibe como parámetros: una matrizen la cual se encuentra la información de los vectores de tiempo de cada modulación, una hipermatriz de conmutaciones en la cual se encuentra la información de los estados de conmutación de cada modulación, un vector de frecuencias el cual indica la posición de la frecuencia dentro de la matriz de tiempos y la hipermatriz de conmutaciones, un vector denominado vector de largos que indica cuantos tiempos y estados tiene cada una de las modulaciones, y por último recibe el valor de la frecuencia que se desea que el inversor genere a la salida.
En la figura 3 se muestra el esquema de simulación, en el cual se observan cuatro etapas fundamentales: como primera fase el convertidor de potencia, enmarcado en el cuadro 1, en donde se muestran los seis puentes $\mathrm{H}$ junto con los transformadores a la salida de cada puente; en el recuadro 2 se observa la etapa de generación de las señales de control; en el recuadro 3 se demarcan los medidores de calidad de la energía y de valores RMS, por último en el cuadro 4 se muestra el motor de inducción.

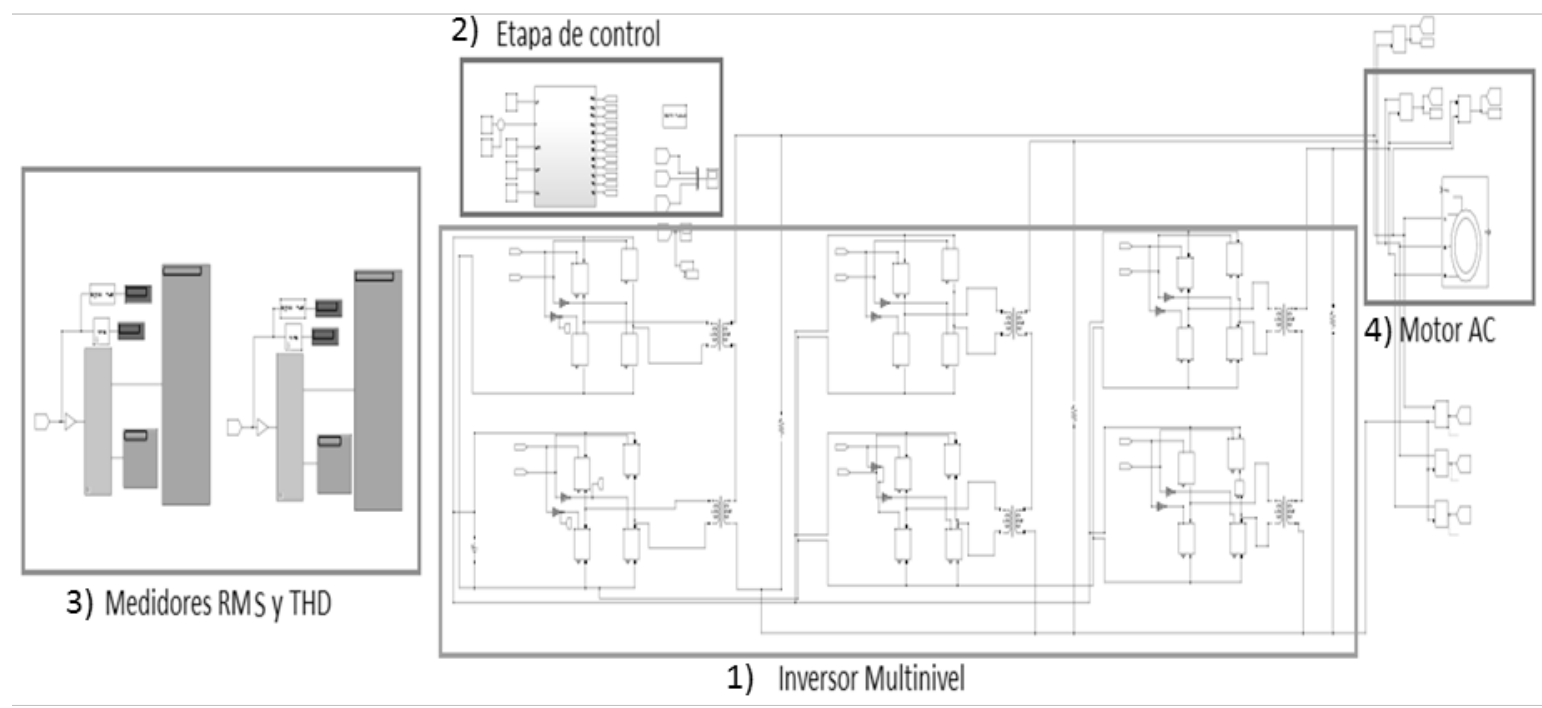

Figura 3. Esquema de simulación.

La ley de control escalar del variador de frecuencia se estableció con modulaciones calculadas con un algoritmo genético, desarrollado por los autores en trabajos previos (Díaz-Rodríguez, Pabón-Fernández, \& Pardo-García, 2015; PabónFernández, Díaz-Rodríguez, \& Pardo-García, 2016).
Este algoritmo permite optimizar el contenido armónico de las tensiones de línea e incluye una variación para poder calcular modulaciones con diferentes tensiones en dependencia de la ley de mando establecida para este trabajo. En la figura 4 se muestra la relación escalar $V$ vs $\mathrm{f}$ establecida. 


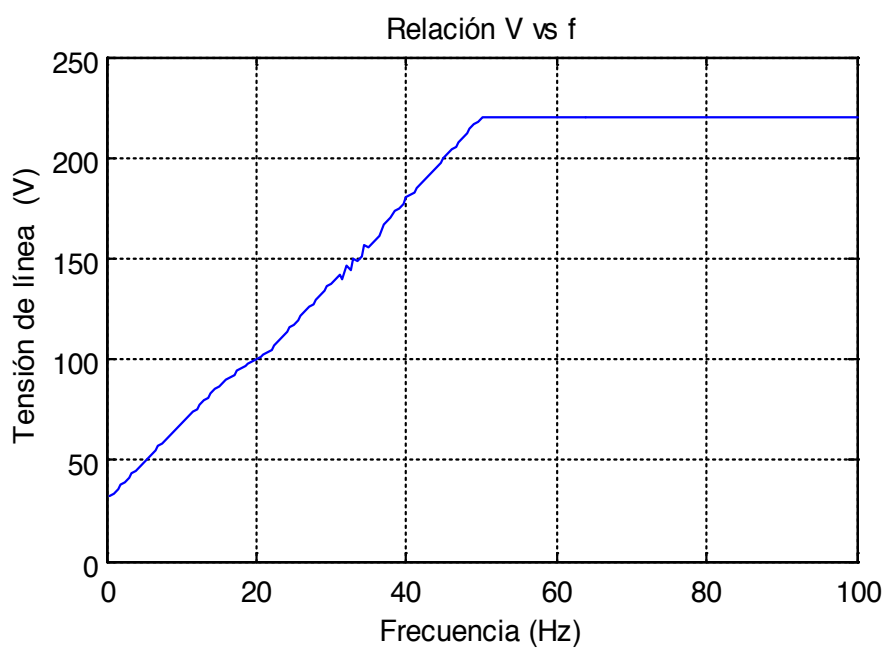

Figura 4. Relación escalar $\mathrm{V}$ vs $\mathrm{f}$ con los valores obtenidos por el algoritmo multiobjetivo.

\section{Resultados y discusión}

A continuación se indican algunos puntos de muestra de la ley escalar para validar el buen contenido armónico que se presenta en las tensiones de alimentación del motor.

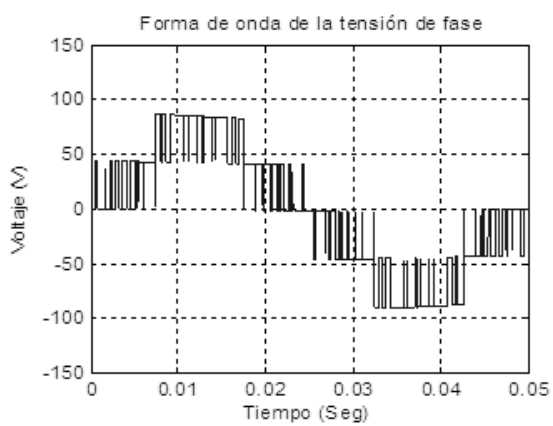

a)

\subsection{Frecuencia de $20 \mathrm{~Hz}$}

Las formas de onda de la tensión de fase y de línea a la salida del variador de frecuencia, se muestran en la figura 5 .

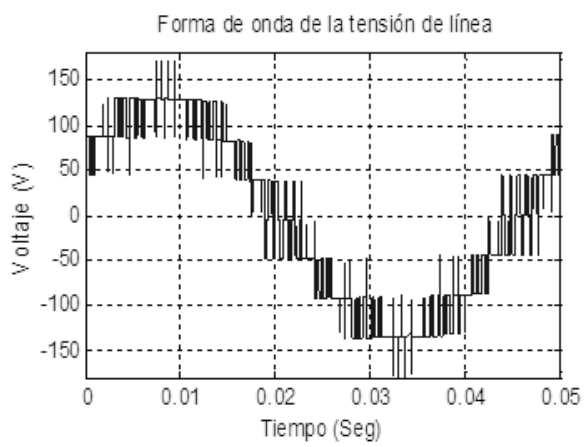

b)

Figura 5. a) Forma de onda de la tensión de fase, b) Forma de onda de la tensión de línea para $20 \mathrm{~Hz}$.

La forma de onda de la tensión de fase se observa en la figura $5 a$, cuya modulación posee cinco niveles en cada fase. En la figura $5 \mathrm{~b}$ se muestra la forma de onda de la tensión de línea, donde se evidencia que el número de niveles aumenta a 9. También se incrementa el número de pulsos en cada nivel, lo cual se debe a la resta entre las tensiones de fase que genera la tensión de línea con más pulsos y más escalones.

En cuanto a los armónicos, la figura 6a presenta el espectro evaluado por Simulink de la tensión de fase, mientras que la figura $6 \mathrm{~b}$ muestra el espectro de la tensión de línea. 


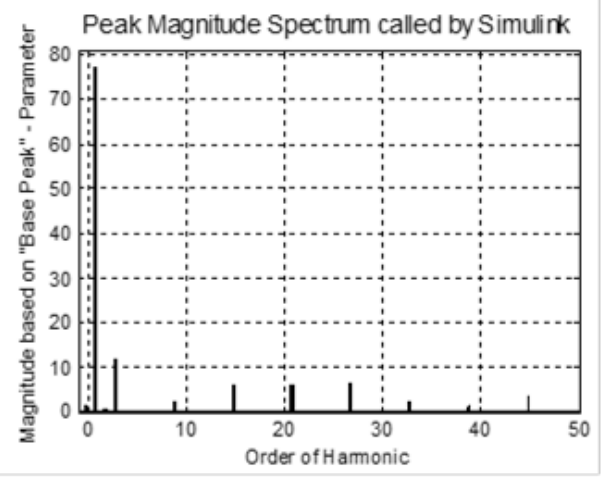

a)

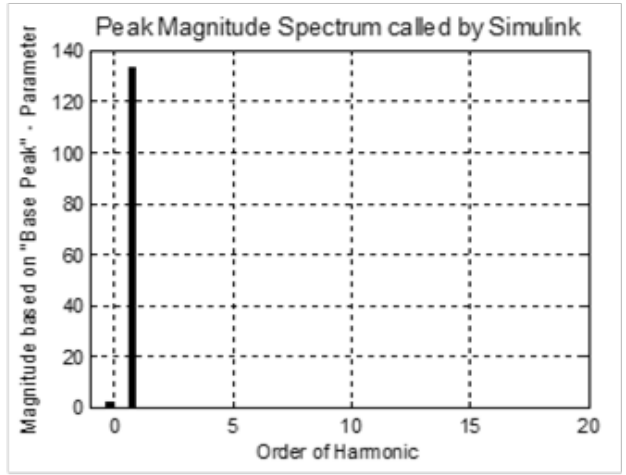

b)

Figura 6. a) Espectro de la tensión de fase, b) Espectro de la tensión de línea.

En el espectro de fase se encuentran los terceros armónicos, esto no es de importancia ya que la optimización se realiza en la tensión de línea y, según el algoritmo de optimización, en la tensión de línea esta presencia de armónicos triplens debe desaparecer. Lo anterior se verifica en la figura $6 \mathrm{~b}$, donde se observa que el espectro armónico

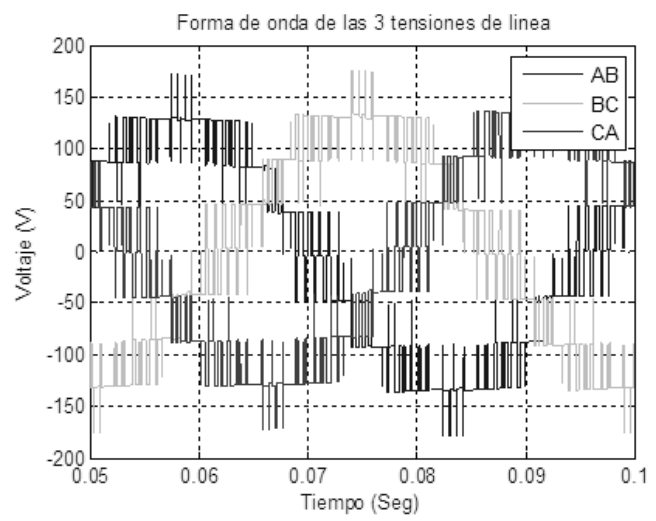

a) de la tensión de línea no tiene componentes armónicas apreciables. Las formas de onda de las tres tensiones de línea se muestran en la figura $7 a$, donde se observa que el sistema es trifásico equilibrado y que las tres tensiones de línea poseen la misma forma de onda.

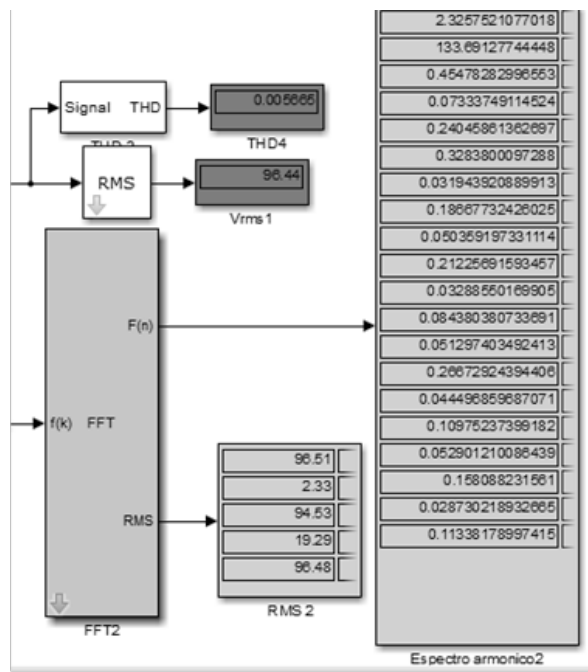

b)

Figura 7. a) Forma de onda del sistema equilibrado trifásico, b) Valor RMS y THD de la tensión de línea para f=20 Hz 
En la Figura $7 \mathrm{~b}$ se observa el contenido armónico de la forma de onda de la tensión de línea, donde el THD es 0,56\% y el valor RMS es de $96,44 \mathrm{~V}$. Así se valida la eficiencia del algoritmo de optimización para variar voltaje y frecuencia, manteniendo el THD de línea en valores muy bajos, cercanos al cero por ciento y cumpliendo a cabalidad con la norma IEEE 519 (Institute of Electrical and Electronics Engineers Std 519[IEEE Std 519], 1992).

\subsection{Frecuencia de $50 \mathrm{~Hz}$}

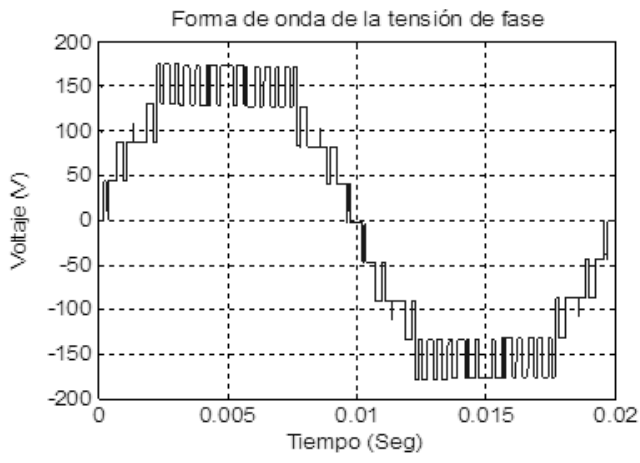

a)
Las formas de onda de la tensión de fase y de línea se muestran en la figura 8 para una frecuencia de $50 \mathrm{~Hz}$, que es la nominal del motor a controlar. La forma de onda de la tensión de fase posee nueve niveles, como se ve en la figura 8a. En la figura $8 \mathrm{~b}$ se observa que, en la tensión de línea, el número de niveles aumenta, junto con el número de pulsos; esto se debe a la diferencia entre las tensiones de fase que genera la tensión de línea.

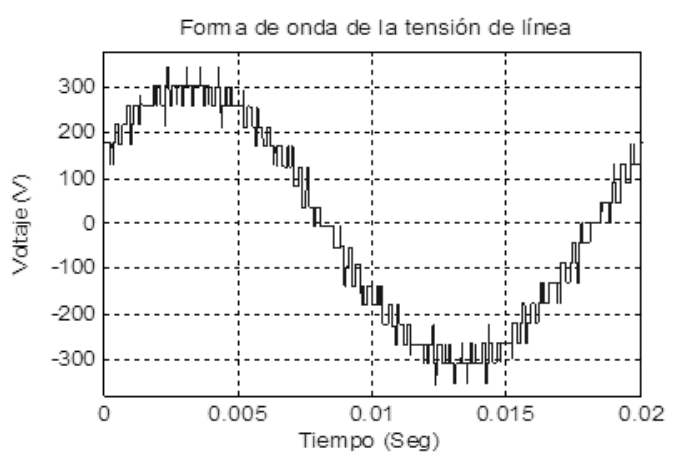

b)

Figura 8. a) Forma de onda de la tensión de fase, b) Forma de onda de la tensión de línea para $50 \mathrm{~Hz}$.

La onda de voltaje de línea tiene 15 escalones. En cuanto a los espectros armónicos, la figura 9a. muestra el espectro evaluado por Simulink de la tensión de fase y la figura 9b muestra el espectro de la tensión de línea.

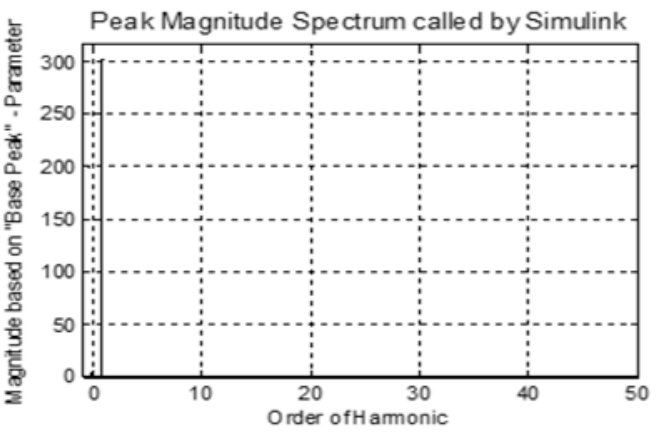

b)

Figura 9. a) Espectro de la tensión de fase, b) Espectro de la tensión de línea 
En el espectro de fase se observa claramente la existencia de los terceros armónicos, esto no es de importancia ya que la optimización se realiza en la tensión de línea. En la figura 9b se observa el espectro de la tensión de línea, en donde se ratifica que los armónicos triplens desaparecieron y el contenido armónico en el orden de los primeros cincuenta componentes es prácticamente nulo.
Las formas de onda de las tres tensiones de línea se muestran en la figura 10a; allí se observa que el sistema trifásico sigue siendo equilibrado y que las tres tensiones de línea poseen la misma forma de onda, además no se presenta la caída de los pulsos como se presentaba en las formas de onda a baja frecuencia.

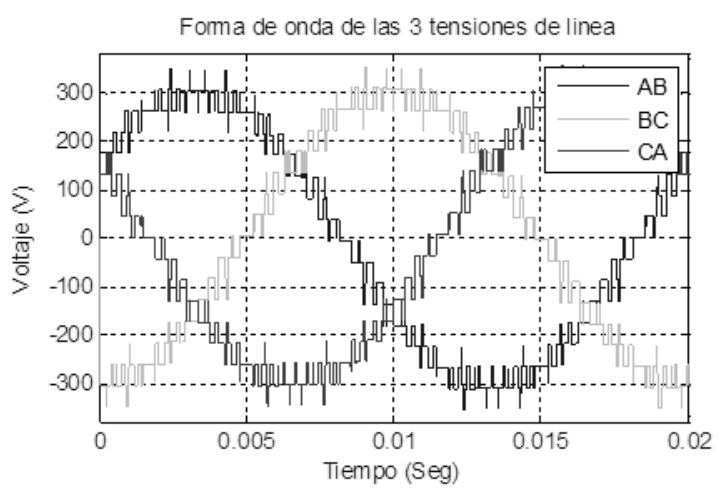

a)

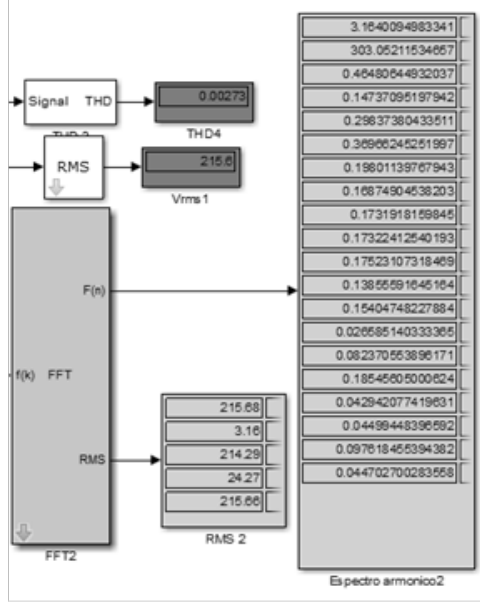

b)

Figura 10. a) Forma de onda del sistema equilibrado trifásico, b) Valor RMS y THD de la tensión de línea para f=50 Hz

En la figura 10b se observa el contenido armónico de la forma de onda de la tensión de línea, este muestra que el THD es $0,273 \%$ y el valor RMS es de 215,6 Voltios. Así se valida la eficiencia del algoritmo de optimización para variar voltaje y frecuencia, manteniendo el THD de línea en valores muy bajos, cercanos al cero por ciento, cumpliendo a cabalidad con la norma IEEE 519.

\subsection{Frecuencia de $60 \mathrm{~Hz}$}

Esta frecuencia es superior a la frecuencia nominal, por tanto el valor RMS de la tensión y la forma de onda serán iguales a las de la modulación de 50 Hz. Las formas de onda de la tensión de fase y de línea se muestran en la figura 11. 


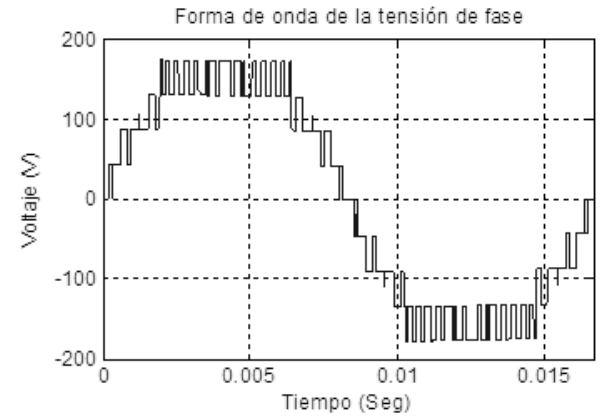

a)

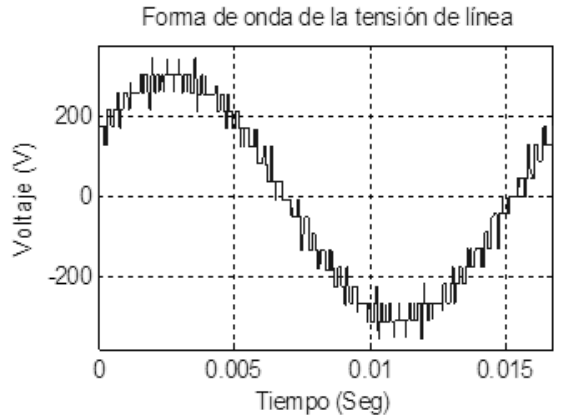

b)

Figura 11. a) Forma de onda de la tensión de fase, b) Forma de onda de la tensión de línea para $60 \mathrm{~Hz}$.

La forma de onda de la tensión de fase se ve en la figura 11 a, donde se observa que la modulación posee los mismos nueve niveles que en la modulación de $50 \mathrm{~Hz}$. En la figura $11 \mathrm{~b}$ se muestra la forma de onda de la tensión de línea, donde el número de niveles es 15 y su forma de onda es igual

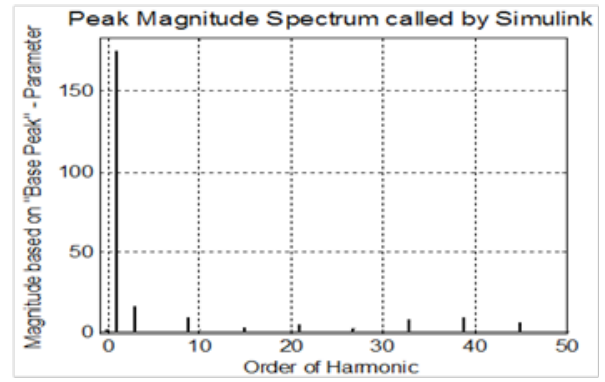

a) a la de $50 \mathrm{~Hz}$. En cuanto a los espectros armónicos, la figura 12a muestra el espectro evaluado por Simulink de la tensión de fase, mientras que la figura $12 \mathrm{~b}$ muestra el espectro de la tensión de línea.

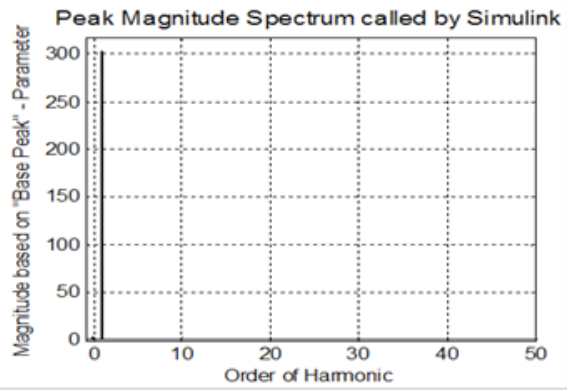

b)

Figura 12. a) Espectro de la tensión de fase, b) Espectro de la tensión de línea.

Las formas de onda de las tres tensiones de línea se muestran en la figura 13a; se observa que el sistema trifásico sigue siendo equilibrado y que las tres tensiones de línea poseen la misma forma de onda. Además, no se presenta la caída de los pulsos como se presentaba en las formas de onda de baja frecuencia. 


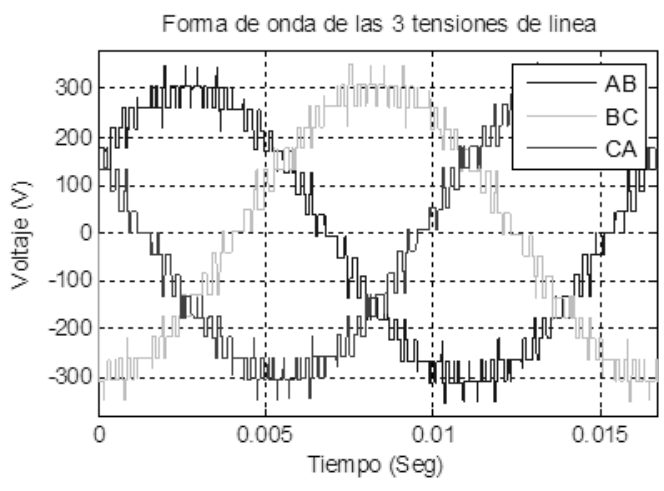

a)

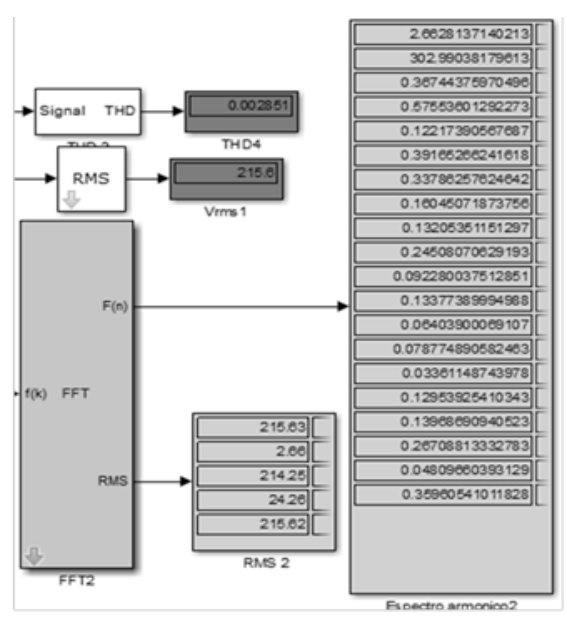

b)

Figura 13. a). Forma de onda del sistema equilibrado trifásico, b) Valor RMS y THD de la tensión de línea para f=60 Hz.

En la figura 13b se observa el contenido armónico de la tensión de línea, donde el THD es 0,273\% y el valor RMS es de 215,6 Voltios. Así se valida la eficiencia del algoritmo de optimización para mantener el voltaje por encima de la frecuencia nominal y variar la frecuencia, manteniendo el THD de línea en valores muy bajos, cercanos al cero por ciento y cumpliendo a cabalidad con la norma IEEE 519.

\subsection{Alimentación del motor con el convertidor}

En primer lugar se asignaron los parámetros del motor de inducción, como se muestra en la figura 14.

\begin{tabular}{|c|c|c|c|}
\hline \multicolumn{4}{|c|}{ 1. Block Parameters: Asynchronous Machine SI Units } \\
\hline \multicolumn{4}{|c|}{ Asynchronous Machine (mask) (link) } \\
\hline \multicolumn{4}{|c|}{$\begin{array}{l}\text { Implements a three-phase asynchronous machine (wound rotor, squirrel } \\
\text { cage or double squirrel cage) modeled in a selectable dq reference frame } \\
\text { (rotor, stator, or synchronous). Stator and rotor windings are connected in } \\
\text { wye to an internal neutral point. }\end{array}$} \\
\hline Configuration & Parameters & Advanced & Load Flow \\
\hline \multicolumn{4}{|l|}{ Preset model: } \\
\hline \multicolumn{4}{|l|}{ No } \\
\hline \multicolumn{4}{|c|}{ Mechanical input: } \\
\hline \multicolumn{4}{|c|}{ Torque Tm } \\
\hline \multicolumn{4}{|l|}{ Rotor type: } \\
\hline \multicolumn{4}{|l|}{ Wound } \\
\hline \multicolumn{4}{|c|}{ Reference frame: } \\
\hline \multicolumn{4}{|l|}{ Rotor } \\
\hline \multicolumn{4}{|c|}{ Measurement output } \\
\hline \multicolumn{4}{|c|}{$\square$ Use signal names to identify bus labels } \\
\hline
\end{tabular}

Figura 14. Asignación de los parámetros del motor de inducción. 
Los valores se asignaron en concordancia con los parámetros nominales del motor de inducción de rotor bobinado, disponibles en el laboratorio de máquinas eléctricas de la universidad de Pamplona, el cual se utilizará en próximas pruebas experimentales.
Como muestra de lo que sucede cuando el variador de frecuencia cambia la frecuencia de alimentación del motor, se realizó una prueba en la cual la frecuencia cambió de 20 a 40 y a $60 \mathrm{~Hz}$ de una manera rápida, es decir en unos cuantos ciclos. Los resultados se presentan en la figura 15.

\section{Tensiones del variador}

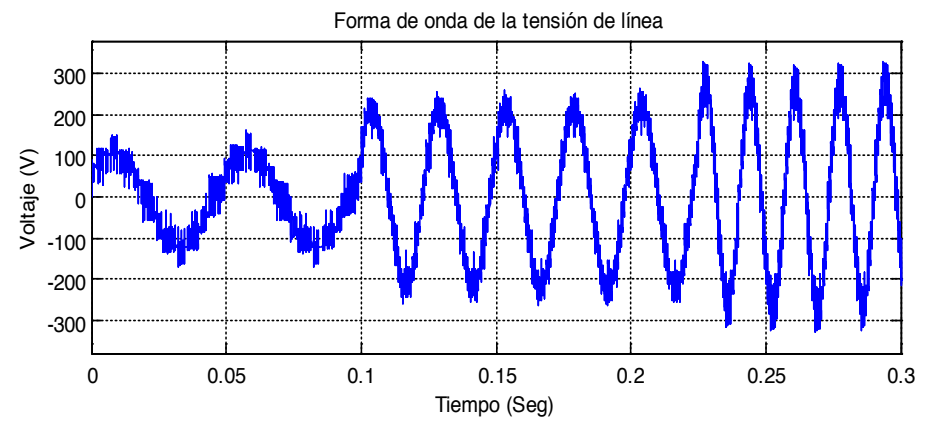

Figura 15. Forma de onda de la tensión de línea frente a variaciones de frecuencia.

En la figura 15 se observa cómo el número de escalones que utiliza el convertidor va variando en términos de aumentar o disminuir la tensión; igualmente se visualiza cómo la frecuencia va cambiando. Esta prueba ratifica el buen funcionamiento del algoritmo de control del convertidor y ratifica el buen funcionamiento del variador.

\section{Corrientes de entrada al motor}

Para la prueba anterior se observaron las corrientes de entrada al motor en los transitorios de cambio de frecuencia. Las corrientes tienen la forma de la figura 16, las cuales no son ondas sinusoidales constantes sino que van cambiando su magnitud; sin embargo, el comportamiento sinusoidal es innegable.

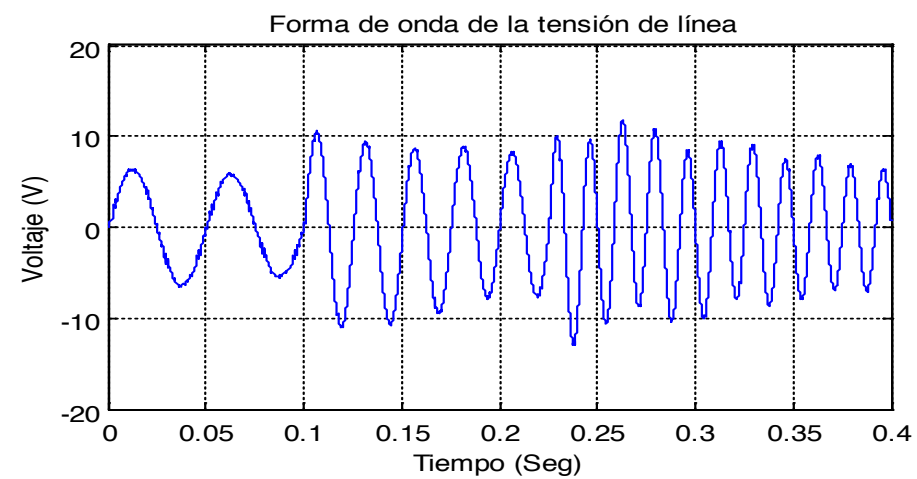

Figura 16. Forma de onda de la corriente de línea frente a variaciones de frecuencia.

En la figura 16 se observa que la corriente, sea cual sea la frecuencia, tiene forma sinusoidal; esto deja entrever que el espectro armónico y que el THD van a ser muy bajos. Para verificar esta condición, 
se alimentó el motor con una frecuencia de $60 \mathrm{~Hz}$, superior a la nominal, y se midieron las variables del motor, incluida la corriente del estator. En la figura 17 se muestra la forma de la corriente durante el arranque y en estado estacionario.

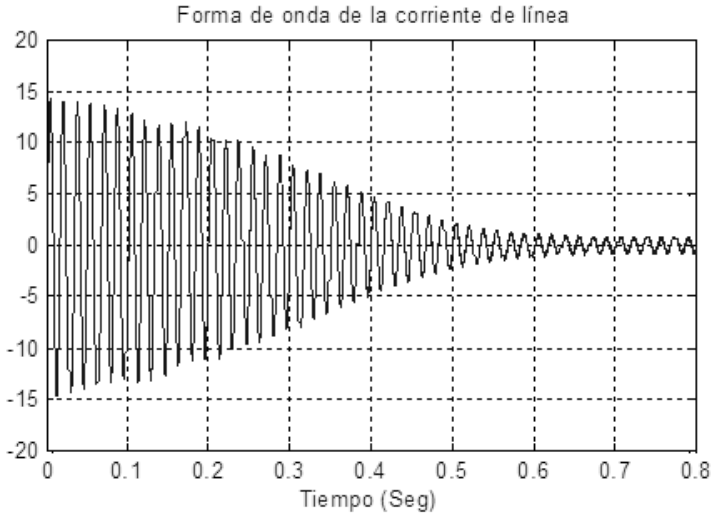

a)

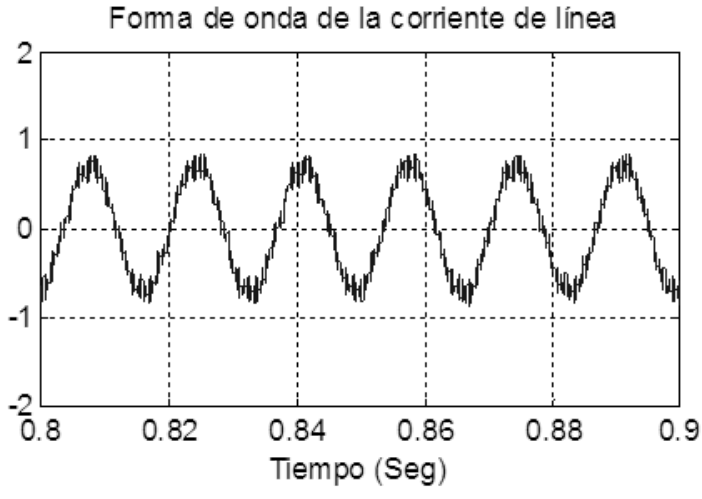

b)

Figura 17. a) Forma de onda de la corriente de línea en arranque, b) Forma de onda en estado estacionario

En la figura $17 \mathrm{~b}$ se muestra la forma de onda de la corriente de estado estacionario, la cual tiene una forma cercana a la sinusoidal; sin embargo, se observan algunas perturbaciones en la corriente. En la figura 18 se muestra el contenido espectral de la forma de onda de la corriente cuando tiene un valor cercano a los 10 A RMS. Se observa que el espectro es prácticamente nulo pero se percibe una pequeña presencia de armónicos de bajo orden, la cual no es muy notoria ya que el THD de la onda de corriente tiene un valor de 1,4\%.

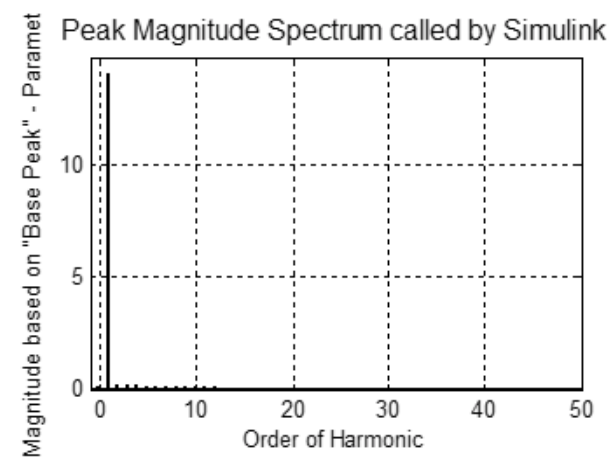

Figura 18. Espectro armónico de la corriente cuando tiene un valor cercano a $10 \mathrm{~A}$.

Igualmente, se muestra en la figura 19 el espectro armónico de la onda de corriente cuando tiene un valor de 0,5 A, que es el valor de estado estacionario. Allí se observa una presencia de armónicos de bajo orden, cuyas magnitudes son más apreciables que las de la corriente de 9,61 A; esto permite deducir que a mayor corriente el filtrado que se genera en 
los devanados de los transformadores y el motor sobre la onda de corriente es mayor.

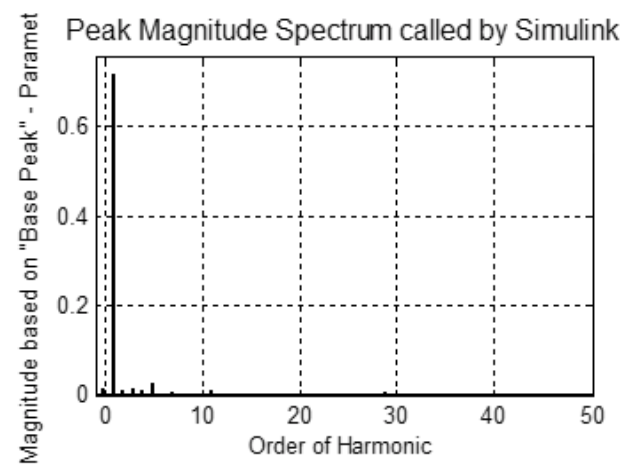

Figura 19. Espectro armónico de la corriente para un valor cercano a $0,5 \mathrm{~A}$.

El THD de la forma de onda de la corriente es de $4,88 \%$, que es un valor bastante alto para lo que se quiere realizar en la optimización. Sin embargo, se espera que en la etapa de implementación esto mejore, ya que los transformadores van a ser realizados bajo una nueva metodología de diseño.

\section{Corrientes rotóricas}

En la figura 20 se ilustra la forma de onda de la corriente rotórica, mostrando que en los momentos de gran inducción la corriente tiene un valor alto; en esos intervalos de tiempo la forma de onda es prácticamente sinusoidal.

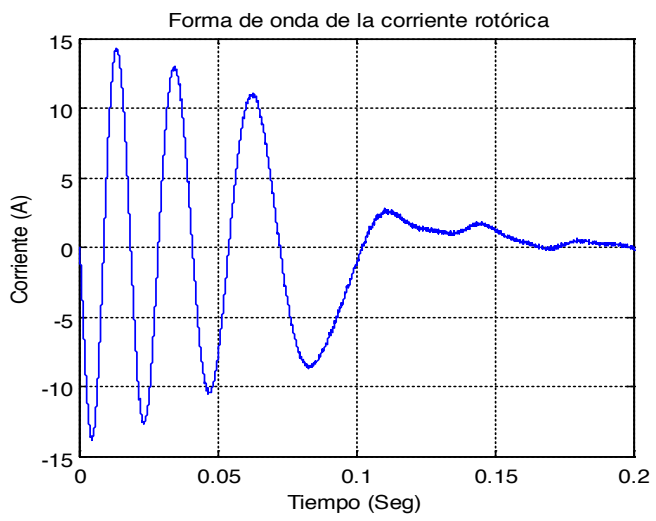

Figura 20. Forma de onda de la corriente rotórica.
La corriente se asemeja bastante a una onda sinusoidal, debido al bajo contenido armónico de la alimentación. Sin embargo, el beneficio de la alimentación de bajo contenido armónico se verá reflejado en el par y en la velocidad del motor.

\section{Velocidad mecánica y par electromagnético}

En la figura 21 se muestra la gráfica de velocidad vs tiempo, donde la velocidad tiene un comportamiento alisado, no existen perturbaciones ni grandes variaciones, tanto en el arranque como en el estado estable; este comportamiento evidencia la ventaja de alimentar el motor de inducción con el convertidor multinivel.

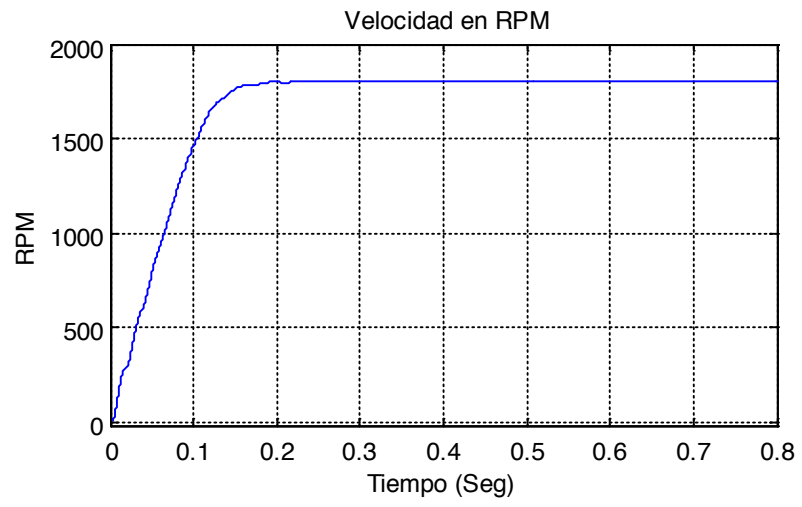

Figura 21. Velocidad mecánica de la máquina en RPM.

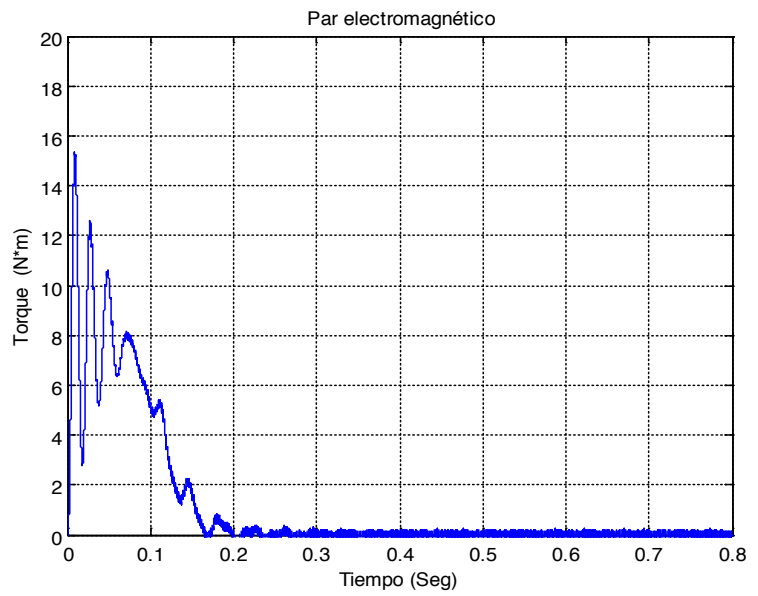

Figura 22. Par electromagnético. 
En la figura 22 se muestra la forma del par electromagnético. Allí se evidencia que el par tiene un comportamiento relativamente suave, debido a que el inversor multinivel, al no inyectar armónicos considerables, no genera pares opuestos lo cual disminuye el rizado en el torque. Este comportamiento es mejor que el presentado en el trabajo de Díaz-Rodríguez, Pabón-Fernández y Anaya (2014).

\section{Conclusiones}

La alimentación del motor de inducción con convertidor de potencia multinivel y optimización de armónicos desarrollado en este trabajo, ofrece ventajas para el motor de inducción, como la reducción de las componentes armónicas de la corriente y tensión. Esto disminuye en teoría el rizado en el par electromagnético, en la velocidad y en las propias corrientes, evitando problemas como pares opuestos, sobrecalentamiento $y$ demás inconvenientes asociados a la distorsión armónica.

En las simulaciones se observa que los escalones de las tensiones de línea no están bien definidos, debido a que se utilizaron modelos de transformadores convencionales. Sin embargo, en la experimentación para construir el prototipo se propone utilizar una nueva metodología de diseño para estas aplicaciones; los transformadores así diseñados se comportan de manera excelente dando resultados muy buenos en trabajos previos (Díaz-Rodríguez, Pabón-Fernández, \& PardoGarcía, 2015).

La simulación permitió validar como primera instancia que las optimizaciones en las tensiones de línea eliminan ostensiblemente también el contenido armónico de las Corrientes. Además, el convertidor multinivel de fuente común trifásico de nueve escalones por fase se puede utilizar como variador de frecuencia. Igualmente, alimentar el motor de inducción con ondas optimizadas tiene beneficios para él, como la disminución en el rizado de las corrientes, en la velocidad y en el par. La simulación también valida la hipótesis presentada en el apartado del modelado matemático, la cual establece que en las fases pueden existir los armónicos triplens en la onda de tensión, pero estos desaparecerán en la línea.

EI THD de las tensiones de línea evidencia la buena optimización realizada por el algoritmo, ya que en todas las pruebas el THD estuvo por debajo del límite establecido por la IEEE 519, de 5\%. EI THD más bajo se presentó en un valor de 0,9\% y el más alto en $3 \%$, que son valores muy buenos en cuanto a optimización se refiere y que supera con creces la banda establecida.

\section{Referencias}

Araque-Gallardo, J. A., Díaz-Rodríguez, J. L., \& Gualdrón-Guerrero, O. (2013). Optimización del THD en un convertidor multinivel monofásico usando algoritmos genéticos. Revista Colombiana de Tecnologías de Avanzada, 1 (21), 60-66. Recuperado de: http://revistas.unipamplona. edu.co/ojs_viceinves/index.php/RCTA/article/ view/297

Araque-Gallardo, J. A., Díaz-Rodríguez, J. L., \& Guerrero, A. (2016). Optimización por recocido simulado de un convertidor multinivel monofásico con modulación PWM sinusoidal de múltiple portadora. Revista Colombiana de Tecnologías de Avanzada, 1 (27), 91-97. Recuperado de: http:// www.unipamplona.edu.co/unipamplona/ portallG/home_40/recursos/05_v25_30/ revista_27/16052016/15.pdf

Barbera, G., Mayer, H.G., \& Issoutibhere, F. (2009). Medición de la emisión armónica en variadores de velocidad y desarrollo de modelos de simulación. En Encuentro Regional Iberoamericano de CIGRE. Encuentro llevado a cabo en Foz de Iguazú, Argentina.

Bermeo, W. L., de Souza, A. B., Fernandes, T. R., Honorio, D. A., Nogueira-dos Reis, L. L., \& Barreto, 
L. H. (2016). Control modo deslizante aplicado en la malla de corriente para una aplicación de una base-DSP para el control de posición de un motor de inducción de jaula de ardilla. Revista Colombiana de Tecnologías de Avanzada, 1 (27), 2632. Recuperado de: http://www.unipamplona.edu. co/unipamplona/portallG/home_40/recursos/05_ v25_30/revista_27/16052016/05.pdf

Castro-Galeano, J.C., Pinto-Salamanca, M. L., \& Amaya-Quitián, M. F. (2014). Diseño y construcción de una Bobina Tesla de $1680 \mathrm{~W}$, para la enseñanza de conceptos básicos en sistemas eléctricos de potencia. Revista de Investigación, Desarrollo e Innovación, 5 (1), 66-74. doi: 10.19053/20278306.3142

Díaz-Rodríguez, J. L., Pabón-Fernández, L. D., \& Torres-Chávez, I. (2012). Análisis comparativo de la distorsión armónica en inversores de potencia. Revista Colombiana de Tecnologías de Avanzada, 1(19), 92-99. Recuperado de: http://revistas. unipamplona.edu.co/ojs_viceinves/index.php/ RCTA/article/view/158

Díaz-Rodríguez, J.L., Pabón-Fernández, L.D., \& Pardo-García, A. (2015). THD improvement of a PWM cascade multilevel power inverters using genetic algorithms as optimization method. WSEAS Transaction on Power Systems, 10, 4654. Recuperado de: http://www.wseas.org/ multimedia/journals/power/2015/a125716-310. pdf

Díaz-Rodríguez, J.L., Pabón-Rodríguez, \& Anaya, Y.C. (2014). Motor de inducción alimentado con convertidor multinivel. Puente, 8 (1), 15-26. Recuperado de: http://puente.upbbga.edu.co/ index.php/revistapuente/article/view/139

Fitzgerald, A.E., Kingsley, C., \& Umans, S.D. (2003). Electric machinery. (6a ed.). Ciudad de México, México: McGraw-Hill.

Institute of Electrical and Electronics Engineers
Std 519 [IEEE Std 519] (1992). IEEE Recommended Practices and Requirements for Harmonic Control in Electrical Power Systems. doi: 10.1109/ IEEESTD.2014.6826459

Malinowski, M., Gopakumar, K., Rodríguez, J., \& Pérez, M. (2010). A survey on cascaded multilevel inverters. IEEE Transactions on Industrial Electronics, 57 (7), 2197-2206. doi: 10.1109/TIE.2009.2030767

Manasa, Balaji-Ramakrishna, S., Madhura, S., \& Mohan, H.M. (2012). Design and simulation of three phase five level and seven level inverter fed induction motor drive with two cascaded h-bridge configuration. International Journal of Electrical and Electronics Engineering, 1 (4), 22315284. Recuperado de: http://www.interscience.in/ IJEEE_Vol1Iss4/Paper6.pdf

Mora, J. (2005). Máquinas eléctricas. Ciudad de México, México: McGraw-Hill.

Mora-Mendoza, E. Y., Sarmiento-Santos, A., \& Casallas-Caicedo, F. M. (2014). Implementación de un sistema de tratamiento con plasma para gases utilizando una celda de descarga de barrera dieléctrica. Revista de Investigación, Desarrollo e Innovación, 5 (1), 56-65. doi: 10.19053/20278306.3141

Pabón-Fernández, L.D., Díaz-Rodríguez, J.L., \& Pardo-García, A. (2016). Total harmonic distortion optimization of the line voltage in single source cascaded multilevel converter. WSEAS Transaction on Systems 60, (8), 110-120. doi: 10.1109/ TIE.2012.2202351

Pinzón-Ardila, O. (2015). Modulación de vector espacial para inversores trifásicos con cuatro ramas de interruptores. Revista Colombiana de Tecnologías de Avanzada, 2 (26), 99-107. Recuperado de: http://www.unipamplona.edu. co/unipamplona/portallG/home_40/recursos/05_ v25_30/revista_26/01052016/15.pdf 
Pradeep, R., \& Jaseerali, E.Y. (Junio de 2015). Induction motor fed from multilevel inverter topology incorporating selective harmonic. $2^{\text {nd }}$ International Conference on Electronics and Communication System. Conferencia llevada a cabo en Combaitore, India. doi: 10.1109/ ECS.2015.7124929

Restrepo-Chaustre, Y., Vecerra-Vargas, J. A., \& Pardo-García, A. (2015). Metodología de detección de fallas de un motor síncrono. Revista Colombiana de Tecnologías de Avanzada, 2 (26), 136-144. Recuperado de: http://www.unipamplona.edu. co/unipamplona/portallG/home_40/recursos/05_ v25_30/revista_26/01052016/20.pdf

Sánchez, M.A. (2009). Calidad de la energía eléctrica. Puebla, México: Instituto Tecnológicoo de Puebla.

Shimi, S.L., Tilak, T., \& Jagdish, K. (2016). Harmonic elimination of a photo-voltaic based cascaded $\mathrm{H}$-bridge multilevel inverter using PSO (particle swarm optimization) for induction motor drive. Energy, 107, 335-346. doi: 10.1016/j. energy.2016.04.033
Taleba, R., Benyoucefa, D., Helaimia, H., \& Boudje, Z. (2015). Cascaded H-bridge asymmetrical sevenlevel inverter using THIPWM for high power induction motor. Energy Procedia, 74, 844-853. doi: 10.1016/j.egypro.2015.07.820

Torres-Barahona, E. A., León-Medina, J. X., \& Torres-Díaz, E. (2012). Sistema de posicionamiento aplicado a la técnica de impresión 3D modelado por deposición fundida. Revista de Investigación, Desarrollo e Innovación, 3 (1), 25-32. Recuperado de: http://revistas.uptc.edu.co/revistas/index.php/ investigacion_duitama/article/view/2135/2091

Vas, P. (1998). Sensorless vector and direct torque control. Oxford, Reino Unido: Clarendon Press.

Yumurtaci M., Vakkas-Üstün, S., Varbak-Neşe, S., \& Çimen, H. (2008). Comparison of output current harmonics of voltage source inverter used different PWM control techniques. WSEAS Transactions on Power Systems 11 (3), 696-703. Recuperado de: http://www.wseas.us/e-library/transactions/ power/2008/31-151.pdf 